\title{
RÉSULTATS DE DEUX ESSAIS DE FERTILISATION SUR PIN MARITIME ADULTE DANS LES LANDES
}

\author{
M. BONNEAU, J. GELPE* et G. ILLY* \\ avec la collaboration technique de B. LEFROU* et P. SARTOLOU* \\ Station de Recherches sur les Sols forestiers et la Fertilisation, \\ Domaine de l'Hermitage, Pierroton \\ Cestas, 33610 Gazinet \\ * Station de Recherches forestières de Bordeaux, \\ Centre national de Recherches forestières, I.N.R.A., \\ Champenoux, 54370 Einville
}

\begin{abstract}
RÉSUMÉ
Sur les podzols landais, au Sud de Bordeaux, deux essais de fertilisation de pin maritime adulte ont été installés en 1962.

Dans le premier, au domaine de Marcheprime (39 ans en 1962), deux traitements, PK et NPK, ont été appliqués. Au cours des 10 ans qu'a duré l'expérience, les doses totales appliquées ont été de $380 \mathrm{~kg}$ de $\mathrm{P}_{2} \mathrm{O}_{5}$ et $212 \mathrm{~kg}$ de $\mathrm{K}_{2} \mathrm{O}$ par ha, appliquées en deux fois en 1962 et 1966, la première fois par enfouissement localisé, la deuxième fois en surface avec un léger travail du sol, et $280 \mathrm{~kg}$ d'azote appliqués en 4 fois en 1963, 1964, 1965 et 1968. L'effet des engrais ne commence à se manifester qu'après 1963. L'accroissement du volume de 1962 à 1971 a été calculé : les deux traitements (NPK et PK) ont un effet significatif à $1 \%$ par rapport au témoin, mais l'effet de l'azote (NPK par rapport à PK) est significatif à $1 \%$ seulement et semble avoir cessé après 1968 . Le gain de production s'établit à $2 \mathrm{~m}^{3} / \mathrm{ha} / \mathrm{an}$ pour le traitement $\mathrm{PK}$ et $3,4 \mathrm{~m}^{3}$ pour NPK, l'accroissement courant moyen du témoin étant de $7,7 \mathrm{~m}^{3} / \mathrm{ha} / \mathrm{an}$.

Dans l'essai de 1'Hermitage (14 ans en 1962), les premières applications d'engrais, effectuées en 1962 par un sous-solage accompagné d'introduction profonde d'engrais phosphatés et potassiques et d'épandage superficiel d'azote, se sont montrées sans effet. Un renouvellement a eu lieu en 1968 par épandage de $120 \mathrm{~kg}$ de $\mathrm{P}_{2} \mathrm{O}_{5}, 120 \mathrm{~kg}$ de $\mathrm{K}_{2} \mathrm{O}$ avec enfouissement léger par passage de rotavator, et $100 \mathrm{~kg}$ d'azote en 1969. L'accroissement en volume pour la période de 1968 à 1971 a été calculé : l'effet du traitement PK est significatif à $5 \%$, celui de NPK à $1 \%$; l'effet de l'azote (NPK par rapport à PK) n'est pas significatif. L'accroissement courant moyen du témoin est de $16,6 \mathrm{~m}^{3} / \mathrm{ha} / \mathrm{an}$, le gain dû au traitement PK de $3,3 \mathrm{~m}^{3}$ et celui dû au traitement NPK de 5,2 $\mathrm{m}^{3}$.

En définitive, il apparaît que les pins maritimes landais réagissent aussi bien que les semis à la fertilisation. L'azote dont l'effet sur les semis est généralement faible, paraît pouvoir jouer ici un rôle intéressant. Des calculs de rentabilité montrent que, après capitalisation à $5 \%$ des sommes investies, la fertilisation peut laisser un bénéfice de l'ordre de 45 à 50 francs par ha et par an.
\end{abstract}

A Marcheprime, le seul des deux peuplements qui ait été gemmé, la fertilisation n'a eu aucun effet sur la production de gemme. 


\section{INTRODUCTION}

L'effet considérable de la fertilisation phosphatée sur le semis de Pin maritime dans les Landes est aujourd'hui très bien connu. Dès que les résultats des premières expérimentations furent clairs, la question se posa tout naturellement de savoir si un effet semblable pouvait être obtenu sur des pins plus âgés, non fertilisés au moment du semis. Dès 1962, la Station de Recherches forestières de Bordeaux mit en place deux essais simples sur des peuplements de 14 et 39 ans. Cependant les techniques d'apport des engrais qui furent employées à ce moment étaient mauvaises et l'effet de la fertilisation n'apparut clairement qu'après renouvellement de celle-ci. Ce n'est donc que 10 ans après le début de ces expérimentations que nous pouvons en publier les résultats.

\section{I. - ESSAI DE MARCHEPRIME}

\section{1. - Situation - Type de station - Peuplement}

L'expérience est située dans la parcelle 19 , série B, de la propriété forestière de la Société Civile du Domaine de Marcheprime. Il s'agit d'une lande à prédominance de Molinia coerulea et Erica Scoparia, avec présence d'Ulex nanus, Ulex europaeus, Erica cinerea. Les sols sont des podzols de morphologie assez variable, tantôt humo-ferrugineux, tantôt ferrugineux, tantôt humiques, avec des horizons B durcis ou non en alios suivant les endroits et situés à une profondeur variant de 65 à $90 \mathrm{~cm}$; les stations les plus fertiles (où la hauteur du peuplement est la plus grande) semblent correspondre à des podzols à horison Bfe profond et non aliotique. La texture est très sableuse, le rapport $\mathrm{C} / \mathrm{N}$ de l'horizon de surface varie de 22 à 46. La teneur en $\mathrm{P}_{2} \mathrm{O}_{5}$ assimilable (Méthode Duchaufour : 2 extractions successives à $\mathrm{H}_{2} \mathrm{SO}_{4} \mathrm{~N} / 250$ et $\mathrm{OHNa} \mathrm{N} / 10$ ) varie de 0,01 à $0,11 \%$ ) en $\mathrm{A}_{0}$; elle s'abaisse dans l'horizon $\mathrm{A}_{2}(0$ à $0,07 \%$, puis se relève en $\mathrm{B}(0,01$ à $0,10 \%$ \% $)$. Le potassium échangeable est peu abondant $(0,02$ à 0,14 m.e. $/ 100 \mathrm{~g})$, avec un minimum en $\mathrm{A}_{2}$, ainsi que le calcium $(0,1$ à 0,4 m.e. dans les horizons minéraux et jusqu'à 2 m.e. en surface). Le pH à l'eau est toujours nettement acide (3,5 environ en surface, 4 à 5 dans les horizons minéraux). Ces sols présentent donc une assez grande variabilité et, tout en restant pauvres, sont d'une qualité plutôt supérieure à la moyenne landaise : certains peuvent même être qualifiés de moyennement pourvus en phosphore, élément qui fait le plus souvent gravement défaut dans les sols de la région.

Le peuplement, issu d'un semis en bandes de 1923, avait 39 ans au début de l'expérience. Le tableau 1 donne ses caractéristiques moyennes par blocs et traitements, au début de l'année 1962. Elles correspondent, suivant les blocs, aux classes 2 et 3 de la table de production de N. Decourt et B. Lemoine, avec cependant un nombre de tiges par ha plus faible que la normale, d'où une surface terrière également plus faible. Le peuplement étant gemmé, les circonférences ont été mesurées à $2 \mathrm{~m} \mathrm{30}$, au-dessus des carres, et même à $3 \mathrm{~m}$ à la fin de l'expérience. Pour donner les surfaces terrières du tableau 1 on a calculé, par extrapolation d'une relation indiquée dans la table de production, les circonférences à $1 \mathrm{~m} 30$. Elles ont également servi à calculer le volume initial donné dans le tableau 2. 
TABLEAU 1 - TABLE 1

Essai de Marcheprime - Caractéristiques initiales au début de 1962 (39 ans)

Marcheprime trial - Initial data at the beginning of year 1962 (age 39)

\begin{tabular}{|c|c|c|c|c|c|c|c|}
\hline $\begin{array}{l}\text { Traitements } \\
\text { Treatments }\end{array}$ & $\begin{array}{c}\text { Hauteur } \\
\text { totale } \\
\text { Total } \\
\text { height } \\
\text { m }\end{array}$ & $\begin{array}{c}\text { Surface } \\
\text { terrière } \\
1 \mathrm{~m} 30 \\
\text { Basal area } \\
\mathrm{m}^{2} / \mathrm{ha}\end{array}$ & $\begin{array}{l}\text { Nombre } \\
\text { de tiges } \\
\text { par ha } \\
\text { Number } \\
\text { of stems }\end{array}$ & $\begin{array}{c}\text { Blocs } \\
\text { Blocks }\end{array}$ & $\begin{array}{c}\text { Hauteur } \\
\text { totale } \\
\text { Total } \\
\text { height } \\
\text { m }\end{array}$ & $\begin{array}{c}\text { Surface } \\
\text { terrière } \\
1 \mathrm{~m} 30 \\
\text { Basal area } \\
\mathrm{m}^{2} / \mathrm{ha}\end{array}$ & $\begin{array}{c}\text { Nombre } \\
\text { de tiges } \\
\text { par ha } \\
\text { Number } \\
\text { of stems }\end{array}$ \\
\hline $\begin{array}{l}\text { T } \ldots \ldots \\
\text { PK } \ldots \ldots \\
\text { NPK } \ldots \\
\text { Moy . . . }\end{array}$ & $\begin{array}{l}18,08 \\
18,26 \\
17,99\end{array}$ & $\begin{array}{l}18,60 \\
18,51 \\
18,73\end{array}$ & $\begin{array}{l}250 \\
235 \\
249\end{array}$ & $\begin{array}{c}\text { I } \\
\text { II } \\
\text { III } \\
\text { IV } \\
\text { V } \\
\text { VI }\end{array}$ & $\begin{array}{l}16,49 \\
17,54 \\
17,73 \\
18,50 \\
18,95 \\
19,43\end{array}$ & $\begin{array}{l}17,00 \\
18,10 \\
18,06 \\
18,96 \\
19,63 \\
19,93\end{array}$ & $\begin{array}{l}255 \\
255 \\
245 \\
231 \\
230 \\
247\end{array}$ \\
\hline
\end{tabular}

\section{2. - Protocole expérimental}

Trois traitements ont été répétés 6 fois en blocs complets mais « éclatés » (les 3 parcelles d'un même bloc ne sont pas forcément mitoyennes). Les parcelles unitaires avaient $50 \times 50 \mathrm{~m}$ dont un carré central de $35 \times 35 \mathrm{~m}$ était seul mesuré. Les blocs ont été constitués par seule référence aux données initiales du peuplement (hauteur, surface terrière, densité) sans tenir compte de la variation des conditions écologiques qui était assez complexe.

Les blocs furent numérotés de I à VI dans l'ordre des peuplements les plus pauvres (les moins hauts et de plus faible surface terrière) aux plus riches.

Les 3 traitements retenus ont été les suivants :

- $\mathrm{T}$ : témoin.

- PK : $180 \mathrm{~kg}$ de $\mathrm{P}_{2} \mathrm{O}_{5}$ et $112 \mathrm{~kg}$ de $\mathrm{K}_{2} \mathrm{O}$ par ha, apportés sous forme d'hyperphosphate Reno (phosphate naturel finement broyé) et de Patentkali; on a donc apporté, en même temps que $\mathbf{P}$ et $\mathrm{K}, 275 \mathrm{~kg}$ de $\mathrm{CaO}$ et $32 \mathrm{~kg}$ de $\mathrm{MgO}$. Ces engrais ont été appliqués en janvier 1962 dans des trous ouverts à la bêche de vigneron, à $30 \mathrm{~cm}$ de profondeur environ, à une densité de 1 trou pour $2 \mathrm{~m}^{2}$. Cette méthode, qui peut paraître aujourd'hui peu réaliste, était motivée par la crainte, d'une part que des engrais, surtout des engrais phosphatés, appliqués en surface, ne soient pas efficaces, et d'autre part qu'un travail du sol généralisé ne lèse gravement le système radiculaire superficiel des pins qui représente, comme chez la plupart des arbres, 70 à $80 \%$ des racines actives.

- NPK : En plus des engrais ci-dessus, $40 \mathrm{~kg}$ d'azote par ha furent appliqués en couverture sous forme d'ammonitrate, au printemps 1963, puis $40 \mathrm{~kg}$ en juillet 1964, puis $100 \mathrm{~kg}$ en février 1965 , sous la même forme.

Ces premières applications d'engrais semblant ne pas avoir d'effet, il fut décidé de les renouveler, mais cette fois en surface, avec enfouissement très léger au cover-crop. Ainsi, furent apportés :

- dans le traitement $\mathrm{PK}$ : $200 \mathrm{~kg}$ de $\mathrm{P}_{2} \mathrm{O}_{5} /$ ha sous forme de scories à $18 \%$ et $100 \mathrm{~kg} \mathrm{de} \mathrm{K}_{2} \mathrm{O} / \mathrm{ha}$ sous forme de sulfate de potassium, en avril 1966. Au total, de 1962 à 1966, ce traitement a donc reçu $380 \mathrm{~kg}$ de $\mathrm{P}_{2} \mathrm{O}_{5}, 212 \mathrm{~kg}$ de $\mathrm{K}_{2} \mathrm{O}, 730 \mathrm{~kg}$ de $\mathrm{CaO}$ et $32 \mathrm{~kg}$ de $\mathrm{MgO}$ par ha.

- dans le traitement NPK : les mêmes engrais que ci-dessus complétés par $100 \mathrm{~kg}$ d'azote (ammonitrate à 33,5\%) en juin 1968. La dose totale d'azote était ainsi portée à $280 \mathrm{~kg} / \mathrm{ha}$. 
TABLEAU 2 - TABLE 2

Essai de Marcheprime - Volume initial en $m^{3} /$ ha au début de l'expérience (début de 1962 - 39 ans)

Marcheprime trial - Initial volume $\left(\mathrm{m}^{3} / \mathrm{ha}\right)$ at the beginning of the test $(1962-39$ years $)$

\begin{tabular}{c|c|c|c|c|c|c|c|c}
\hline Blocs & I & II & III & IV & V & VI & Moy \\
\hline Traitements & & & & & & & & \\
T & & & & & & & & \\
PK & 128 & 140 & 135 & 150 & 157 & 166 & 146 \\
NPK & 119 & 139 & 140 & 146 & 164 & 174 & 147 \\
Moy. & 122 & 138 & 139 & 151 & 160 & 167 & 146 \\
\hline
\end{tabular}

\section{3. - Résultats}

Des mesures de circonférences ont été effectuées à la fin de 1963, 1965, 1968 et 1971. Les résultats de l'essai ont été suivis essentiellement sur le plan de l'accroissement des surfaces terrières à $2 \mathrm{~m} 30$ (tableau 3). A cause de l'élévation progressive des carres, les circonférences ont été mesurées, en 1971, à $3 \mathrm{~m}$ de hauteur. Par calcul, on a rétabli les mesures à $2 \mathrm{~m} 30$.

\section{TABLEAU 3 - TABLE 3}

Essai de Marcheprime - Évolution et accroissement des surfaces terrières à $2 \mathrm{~m} 30$

- Entre parenthèses, les valeurs relatives des accroissements de surface terrière par rapport au témoin.

Marcheprime trial - Basal area (at $2,30 \mathrm{~m}$ level) evolution and increment

- The relative values of basal area increments versus check plots are in brackets.

\begin{tabular}{|c|c|c|c|c|c|c|c|c|c|}
\hline \multirow{2}{*}{$\begin{array}{l}\text { Traitements } \\
\text { Treatments }\end{array}$} & \multicolumn{5}{|c|}{$\begin{array}{c}\text { Surfaces terrières } \mathrm{m}^{2} / \mathrm{ha} \\
\text { Basal areas }\end{array}$} & \multicolumn{4}{|c|}{$\begin{array}{c}\text { Accroissement de } \mathrm{S} . \mathrm{T} . \mathrm{dm}^{2} / \mathrm{ha} / \mathrm{an} \\
\text { pour les périodes suivantes : } \\
\text { B.A. increment } \\
\text { for the following periods : }\end{array}$} \\
\hline & $\begin{array}{l}1962 \\
\text { (début) } \\
39 \text { ans }\end{array}$ & $\begin{array}{l}1963 \\
\text { (fin) } \\
41 \text { ans }\end{array}$ & $\begin{array}{l}1965 \\
\text { (fin) } \\
43 \text { ans }\end{array}$ & $\begin{array}{l}1968 \\
\text { (fin) } \\
46 \text { ans }\end{array}$ & $\begin{array}{l}1971 \\
\text { (fin) } \\
49 \text { ans }\end{array}$ & $\begin{array}{l}1962- \\
1963\end{array}$ & $\begin{array}{l}1963- \\
1965\end{array}$ & $\begin{array}{l}1965- \\
1968\end{array}$ & $\begin{array}{c}1968- \\
1971\end{array}$ \\
\hline $\begin{array}{l}\mathrm{T} \ldots \ldots \\
\mathrm{PK} \ldots \cdots \\
\mathrm{NPK}\end{array}$ & $\begin{array}{l}16,42 \\
16,22 \\
16,50\end{array}$ & $\begin{array}{l}17,51 \\
17,38 \\
17,72\end{array}$ & $\begin{array}{l}18,78 \\
18,84 \\
19,33\end{array}$ & $\begin{array}{l}20,35 \\
21,14 \\
21,90\end{array}$ & $\begin{array}{l}21,72 \\
23,31 \\
24,37\end{array}$ & $\begin{array}{l}54(100) \\
58(106) \\
61(111)\end{array}$ & $\begin{array}{l}63(100) \\
73(116) \\
80(127)\end{array}$ & $\begin{array}{l}52(100) \\
76(146) \\
85(163)\end{array}$ & $\begin{array}{l}45(100) \\
72(156) \\
82(178)\end{array}$ \\
\hline \multicolumn{6}{|c|}{$\begin{array}{l}\text { F calculé - calculated F value } \\
\text { ppds à } \%-5 \% \text { L.S.D. } \\
\text { à } 1 \% \\
\text { à } 1 \%\end{array}$} & N.S. & $\begin{array}{l}12,73 \\
7,5 \\
10,7 \\
15,5\end{array}$ & $\begin{array}{c}57,16 \\
7,1 \\
10,1 \\
14,6\end{array}$ & $\begin{array}{l}10,80 \\
18,3 \\
26,0 \\
37,3\end{array}$ \\
\hline \multicolumn{6}{|c|}{ 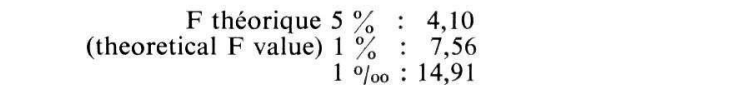 } & & & & \\
\hline
\end{tabular}


L'effet de la fertilisation n'est significatif qu'après 1963; il se renforce pendant la période 1965-68, donc immédiatement après le renouvellement de l'apport d'engrais, puis s'amoindrit à nouveau tout en restant très positif, pour la période 1968-1971. Plus exactement, l'accroissement relatif en surface terrière, par rapport au témoin, semble se renforcer pendant cette période, mais une plus forte variabilité entraîne une baisse de la signification de l'effet traitement.

Le traitement PK est significatif à $1 \%$ ou presque depuis 1963 ; l'effet de NPK atteint presque toujours le seuil de signification à $1 \%$. Par contre, l'effet de l'azote (comparaison de NPK et PK) n'est significatif qu'à $5 \%$ pour la période 1963-65, presque à $1 \%$ en 1965-68 et plus du tout en 1968-71. Dans l'ensemble de la période 1967-1971 son effet est significatif à $5 \%$ et presque à $1 \%$.

Nous avons calculé l'accroissement en volume du début de 1962 à la fin de 1971, donc pendant 10 saisons de végétation. Le volume initial a été calculé à partir des surfaces terrières ramenées à $1 \mathrm{~m} 30$ et des hauteurs moyennes, en appliquant l'abaque donné dans la table de production. Le volume final a été obtenu par mesure de la circonférence à mihauteur et de la hauteur bois fort mesurées arbre par arbre (calcul effectué par B. LEMOINE). Les caractéristiques finales du peuplement et les accroissements en volume sont donnés dans les tableaux 4 et 5 . L'effet des traitements PK et NPK est très hautement significatif et l'effet de l'azote est significatif à $1 \%$.

TABLEAU 4 TABLE 4

Essai de Marcheprime - Caractéristiques finales à la fin de 1971 (49 ans)

Marcheprime trial - Final date at the end of 1971 (49 years)

\begin{tabular}{|c|c|c|c|c|c|}
\hline $\begin{array}{l}\text { Traitements } \\
\text { Treatments }\end{array}$ & $\begin{array}{c}\text { S.T. à } 1 \mathrm{~m} 30 \\
1 \mathrm{~m} 30 \mathrm{~B} . \mathrm{A} . \\
\mathrm{m}^{2} / \mathrm{ha}\end{array}$ & $\begin{array}{l}\text { Hauteur } \\
\text { bois fort } \\
\mathrm{m}\end{array}$ & Blocs & $\begin{array}{c}\mathrm{S} . \mathrm{T} . \text { à } 1 \mathrm{~m} 30 \\
\begin{array}{c}1 \mathrm{~m} 30 \mathrm{~B} . \mathrm{A} . \\
\mathrm{m}^{2} / \mathrm{ha}\end{array}\end{array}$ & $\begin{array}{l}\text { Hauteur } \\
\text { bois fort } \\
\mathrm{m}\end{array}$ \\
\hline $\begin{array}{l}\text { T } \ldots \ldots \ldots \ldots \\
\text { PK } \\
\text { NPK } \ldots \ldots \ldots\end{array}$ & $\begin{array}{l}21,72 \\
23,31 \\
24,37\end{array}$ & $\begin{array}{l}18,1 \\
18,7 \\
18,7\end{array}$ & $\begin{array}{c}\text { I } \\
\text { II } \\
\text { III } \\
\text { IV } \\
\text { V } \\
\text { VI }\end{array}$ & $\begin{array}{l}22,30 \\
22,66 \\
23,05 \\
23,20 \\
24,06 \\
23,54\end{array}$ & $\begin{array}{l}17,9 \\
17,7 \\
18,4 \\
18,7 \\
19,0 \\
19,4\end{array}$ \\
\hline
\end{tabular}

La moyenne des accroissements courants pour ces 10 années est de 7,7 $\mathrm{m}^{3}$ par ha et par an pour le témoin, 9,7 $\mathrm{m}^{3}$ pour le traitement PK et $11,1 \mathrm{~m}^{3}$ pour NPK, soit des gains par rapport au peuplement non fertilisé de $2 \mathrm{~m}^{3} / \mathrm{ha} /$ an pour la fertilisation phospho-potassique et $3,4 \mathrm{~m}^{3} /$ ha/an pour la fertilisation complète NPK.

Si l'on tient compte du fait qu'aucun effet significatif ne s'était manifesté à la fin de 1963, les gains réels dus à la fertilisation sont même probablement supérieurs et se situent dans une fourchette de 2 à $2,5 \mathrm{~m}^{3}$ pour PK et 3,4 à $4,3 \mathrm{~m}^{3}$ pour NPK.

Il est curieux de constater que les accroissements diffèrent assez peu d'un bloc à l'autre, 
et même tendent à être plus élevés dans le bloc 1 qui était classé comme le moins fertile au début de l'expérience. L'hypothèse d'un épuisement général du sol en fin de révolution serait à examiner.

\section{TABLEAU 5 - TABLE 5}

Essai de Marcheprime - Volumes en fin d'expérience, en 1971 (49 ans) et accroissements en volume de 1962 à 1971, en $\mathrm{m}^{3} / \mathrm{ha}$.

Marcheprime trial - Volumes at the end of the trial, in 1971 (49 years) and volume increments from 1962 to $1971\left(\mathrm{~m}^{3} / \mathrm{ha}\right)$.

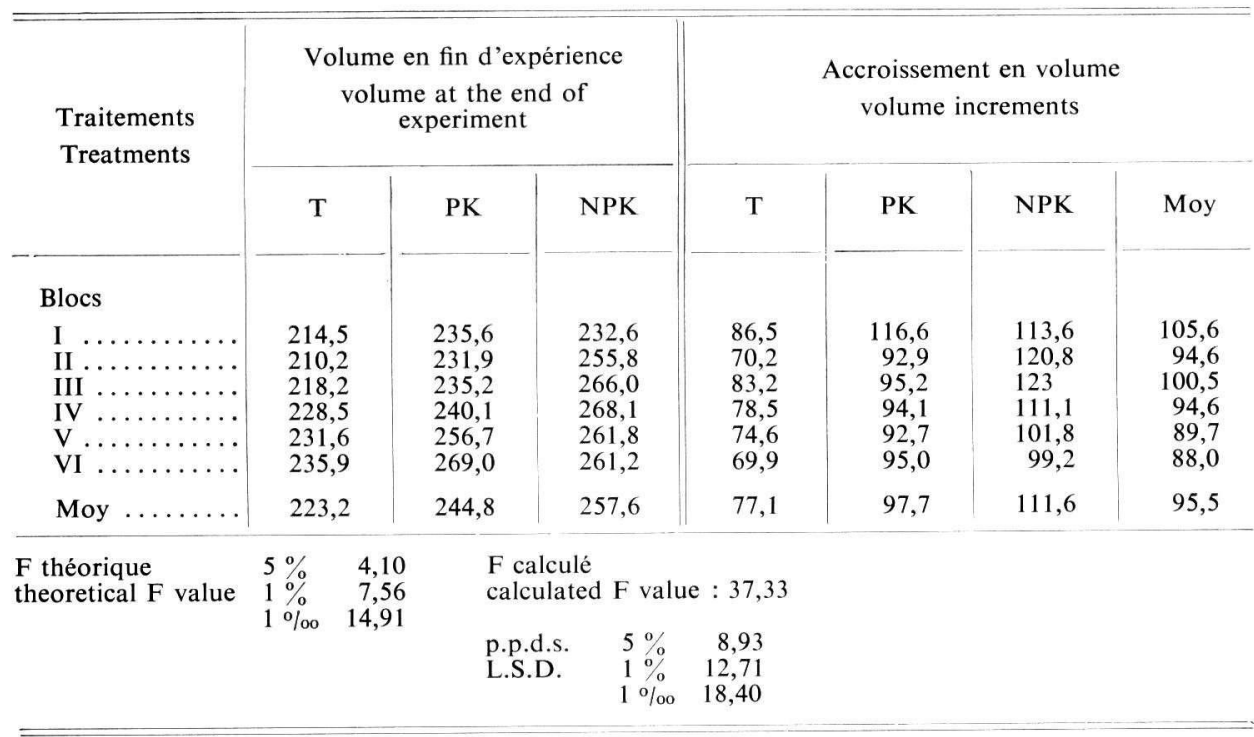

La production de gemme a été mesurée régulièrement de 1962 à 1968 . Les traitements se sont toujours révélés sans effet sur elle. Par contre, elle diffère beaucoup entre blocs comme le montre le tableau 6. L'écart de production entre blocs diminue d'ailleurs de 1962

TABLEAU 6 - TABLE 6

Essai de Marcheprime - Production de gemme (barras exclu) en $\mathrm{kg} / \mathrm{ha}$ cheprime trial - Gum production in $\mathrm{kg} / \mathrm{ha}$ (no including the rosin left on the face).

\begin{tabular}{|c|c|c|c|c|c|c|c|}
\hline Blocs & $\begin{array}{c}1962 \\
39 \text { ans }\end{array}$ & $\begin{array}{c}1963 \\
40 \text { ans }\end{array}$ & $\begin{array}{c}1964 \\
41 \text { ans }\end{array}$ & $\begin{array}{c}1965 \\
42 \text { ans }\end{array}$ & $\begin{array}{c}1966 \\
43 \text { ans }\end{array}$ & $\begin{array}{c}1967 \\
44 \text { ans }\end{array}$ & $\begin{array}{c}1968 \\
45 \text { ans }\end{array}$ \\
\hline $\begin{array}{l}\text { I } \ldots \ldots \\
\text { II } \ldots \ldots \\
\text { III } \ldots \ldots \\
\text { IV } \ldots \ldots \\
\text { V } \ldots \ldots \\
\text { VI } \ldots \ldots\end{array}$ & $\begin{array}{l}188 \\
276 \\
324 \\
290 \\
353 \\
378\end{array}$ & $\begin{array}{l}246 \\
312 \\
374 \\
354 \\
362 \\
412\end{array}$ & $\begin{array}{l}226 \\
291 \\
356 \\
312 \\
394 \\
414\end{array}$ & $\begin{array}{l}364 \\
421 \\
467 \\
450 \\
521 \\
601\end{array}$ & $\begin{array}{l}480 \\
525 \\
560 \\
570 \\
549 \\
582\end{array}$ & $\begin{array}{l}550 \\
588 \\
610 \\
621 \\
646 \\
667\end{array}$ & $\begin{array}{l}605 \\
622 \\
650 \\
648 \\
671 \\
701\end{array}$ \\
\hline
\end{tabular}


à 1968 et on retrouve donc là une observation analogue à celle qui a été faite ci-dessus pour les accroissements en volume. On doit également conclure que la production de gemme ne dépend pas de l'alimentation en éléments majeurs, puisque la fertilisation n'a aucun effet, et qu'elle obéit à un facteur de fertilité différent; celui-ci pourrait être l'alimentation en eau : on se souvient en effet que les blocs les plus fertiles avaient un horizon B profond et non induré. On peut aussi envisager qu'il s'agit d'un élément minéral autre que ceux apportés par la fertilisation.

\section{II. - ESSAI DE L'HERMITAGE (parcelle R)}

\section{1. - Situation - Type de station - Peuplement}

Le Domaine de l'Hermitage, à $20 \mathrm{~km}$ au Sud-Ouest de Bordeaux, appartient à l'État et est occupé par la Station de Recherches forestières de Bordeaux. La parcelle R a été semée en bandes en 1948. Les sols, très sableux, sont des sols podzoliques hydromorphes, allant dans certaines parties jusqu'au podzol humique à alios organique. Les rapports $\mathrm{C} / \mathrm{N}$, non mesurés en $A_{1}$, varient en $A_{0}$ de 21 à 27 et se maintiennent à des valeurs élevées (17 à 26) dans les horizons $A_{2}$ infiltrés de matière organique, descendant exceptionnellement à 13 dans l'un des profils. La teneur en $\mathrm{P}_{2} \mathrm{O}_{5}$ varie de 0,01 à $0,08 \%$ dans les horizons $\mathrm{A}_{2}$ et $\mathrm{B}$ avec une majorité de valeurs très basses de l'ordre de 0,01 à $0,02 \%$, les valeurs les plus élevées se rencontrant en $B$ et au voisinage de $A_{1}$. La teneur en potassium est partout très basse $(0,02$ m.e. pour $100 \mathrm{~g})$; le calcium atteint en $A_{1}$ des valeurs proches de 1 m.e pour $100 \mathrm{~g}$, par concentration biologique, mais ne dépasse que rarement $0,2 \mathrm{~m}$.e dans les horizons inférieurs. La fertilité semble du même ordre qu'à Marcheprime pour le phosphore, le calcium et l'azote, mais est nettement plus basse pour le potassium. Molinia coerulea et Erica Scoparia forment l'essentiel de la végétation spontanée. La callune est parfois présente ; Ulex nanus et Ulex europaeus sont plus rares qu'à Marcheprime.

Les caractéristiques initiales du peuplement, au moment où fut entreprise l'expérience au printemps 1962, sont données au tableau 7. Le peuplement se range suivant les blocs

TABLEAU 7 - TABLE 7

Essai de l'Hermitage - Données initiales en juin 1962 (14 ans)).

L'Hermitage trial - Initial data in june 1962 (14 years).

\begin{tabular}{|c|c|c|c|c|c|c|c|}
\hline Traitements & $\begin{array}{c}\text { Hauteur } \\
\text { dominante } \\
\text { dominant } \\
\text { Height } \\
\text { m }\end{array}$ & $\begin{array}{c}\text { Surface } \\
\text { terrière } \\
1 \mathrm{~m} 30 \\
\text { Basal area } \\
\mathrm{m}^{2} / \mathrm{ha}\end{array}$ & $\begin{array}{l}\text { Nombre } \\
\text { de tiges } \\
\text { par ha } \\
\text { Number } \\
\text { of stems }\end{array}$ & Blocs & $\begin{array}{c}\text { Hauteur } \\
\text { dominante } \\
\text { dominant } \\
\text { Height }\end{array}$ & $\begin{array}{c}\text { Surface } \\
\text { terrière } \\
1 \mathrm{~m} 30 \\
\text { Basal area } \\
\mathrm{m}^{2} / \mathrm{ha}\end{array}$ & $\begin{array}{c}\text { Nombre } \\
\text { de tiges } \\
\text { par ha } \\
\text { Number } \\
\text { of stems }\end{array}$ \\
\hline $\begin{array}{l}\text { T } \ldots \ldots \\
\text { TR } \ldots \ldots \\
\text { PK } \ldots . \\
\text { NPK } \ldots\end{array}$ & $\begin{array}{l}10,1 \\
10,3 \\
10,4 \\
10,4\end{array}$ & $\begin{array}{l}12,9 \\
12,5 \\
12,4 \\
12,2\end{array}$ & $\begin{array}{l}830 \\
875 \\
800 \\
890\end{array}$ & $\begin{array}{l}\text { I } \\
\text { II } \\
\text { III } \\
\text { IV }\end{array}$ & $\begin{array}{r}9,7 \\
10,1 \\
10,2 \\
10,5\end{array}$ & $\begin{array}{l}10,2 \\
11,0 \\
12,4 \\
13,4\end{array}$ & $\begin{array}{l}766 \\
823 \\
869 \\
891\end{array}$ \\
\hline Moy ..... & 10,3 & 12,5 & 849 & V & 10,8 & 15,4 & 960 \\
\hline
\end{tabular}


dans les classes 1 et 2 de la table de production. Le nombre de tiges est cependant nettement plus faible que ne l'indique la table, ainsi que, dans une moindre mesure, les surfaces terrières. Ceci est dû au grand écartement des bandes de semis $(5 \mathrm{~m}$ d'axe en axe pour $1 \mathrm{~m}$ de largeur semée). Les classes de fertilité, meilleures qu'à Marcheprime, ne correspondent donc pas très bien à l'appréciation faite à partir des analyses de sol; ces dernières sont toujours incertaines et ne prennent pas en considération un facteur important : l'alimentation en eau.

\section{2. - Protocole expérimental}

On a appliqué 4 traitements répétés 5 fois suivant la méthode des blocs complets. Comme dans l'essai de Marcheprime, nous avions le souci d'introduire les engrais phosphatés en profondeur et il fut décidé de les appliquer au "Revigor », sous-soleuse permettant une distribution d'engrais en profondeur par une goulotte située en arrière de la dent de sous-solage. Un témoin sous-solé, mais ne recevant pas d'engrais, devait donc être ajouté au témoin pour le cas où le sous-solage aurait par lui-même une action (ce qui, en définitive, ne s'est pas produit). Les 4 traitements appliqués furent donc les suivants :

- $\mathrm{T}$ : témoin;

— TR : témoin sous-solé de la même manière que les traitements suivants, mais sans apport d'engrais;

- PK : deux passages de « Revigor » au milieu de l'interbande de $5 \mathrm{~m}$ ont enfoui successivement $250 \mathrm{~g}$ d'hyperphosphate Reno à $29,5 \%$ et $104 \mathrm{~g}$ de sulfate de potassium à $48 \%$ par mètre linéaire, soit $150 \mathrm{~kg}$ de $\mathrm{P}_{2} \mathrm{O}_{5}$ et $100 \mathrm{~kg}$ de $\mathrm{K}_{2} \mathrm{O}$ par ha; l'épandage a eu lieu à la mi-novembre 1962.

- NPK : même traitement que ci-dessus complété par un épandage superficiel de $40 \mathrm{~kg}$ d'azote par ha, en juillet 1964, sous forme d'ammonitrate à $33,3 \%$, puis de $100 \mathrm{~kg}$ d'azote par ha en mars 1965.

Devant l'inefficacité de ces traitements, due très certainement à un mode d'emploi inadéquat des engrais phosphatés et potassiques, de nouveaux apports furent effectués en 1968 et 1969 :

- PK : $120 \mathrm{~kg} \mathrm{de} \mathrm{P}_{2} \mathrm{O}_{5} /$ ha sous forme de scories à $18 \%$ et $120 \mathrm{~kg} \mathrm{de} \mathrm{K}_{2} \mathrm{O}$ sous forme de sulfate de potasse à $50 \%$, épandus en surface dans les interbandes puis enfouis par un passage de Rotavator en juin 1968.

- NPK : même traitement que ci-dessus complété par $100 \mathrm{~kg}$ d'azote par ha en surface, sous forme d'ammonitrate, en juin 1969.

Les doses totales sont donc de $270 \mathrm{~kg}$ de $\mathrm{P}_{2} \mathrm{O}_{5}, 220 \mathrm{~kg}$ de $\mathrm{K}_{2} \mathrm{O}$ et $240 \mathrm{~kg}$ de $\mathrm{N}$ par ha.

Les parcelles unitaires ont $50 \times 35 \mathrm{~m}$, sont entièrement fertilisées, mais seul un rectangle interne de $35 \times 25 \mathrm{~m}$ est mesuré.

\section{3. - Résultats}

Des mesures de circonférence ont été effectuées en 1963, 1965 et 1968 sans que l'on puisse constater aucun effet. Le tableau 8 donne d'ailleurs les accroissements de surface terrière de 1962 à 1968 : ils sont pratiquement identiques dans tous les traitements. Par contre, les mesures de 1970 et 1971 montrèrent un effet certain, dû vraisemblablement au deuxième épandage d'engrais. Une éclaircie ayant eu lieu en 1968, nous donnons également, de manière succincte, la nouvelle situation de départ à la fin de 1968, après éclaircie, et la situation à la fin de 1971 (tableau 8). 


\section{TABLEAU 8 - TABLE 8}

Essai de l'Hermitage - Accroissement de surface terrière de 1962 à 1968 avant éclaircie (14 à 20 ans) Situation en septembre 1968 après éclaircie et en novembre 1971 ( 23 ans).

L'Hermitage trial - Basal area increment from 1962 to 1968 (age 14 to 20 years) before thinning. Measurements in september 1968 after thinning and in november 1971 (23 years).

\begin{tabular}{|c|c|c|c|c|c|c|}
\hline \multirow[b]{2}{*}{ Traitements } & \multirow{2}{*}{$\begin{array}{c}\text { Accroissement } \\
\text { de S.T. } \\
\text { Basal area } \\
\text { increment } \\
1962-68 \\
\mathrm{~m}^{2} / \mathrm{ha}\end{array}$} & \multicolumn{2}{|c|}{ Septembre 1968} & \multicolumn{2}{|c|}{ Novembre 1971} & \multirow[b]{2}{*}{$\begin{array}{c}\text { Nombre } \\
\text { de tiges } \\
\text { Number } \\
\text { of stems }\end{array}$} \\
\hline & & $\begin{array}{c}\text { Surface } \\
\text { terrière } \\
\text { Basal area } \\
\mathrm{m}^{2} / \mathrm{ha}\end{array}$ & $\begin{array}{c}\text { Hauteur } \\
\text { dominante } \\
\text { dominant } \\
\text { Height } \\
\text { m }\end{array}$ & $\begin{array}{c}\text { Surface } \\
\text { terrière } \\
\text { Basal area } \\
\mathrm{m}^{2} / \mathrm{ha}\end{array}$ & $\begin{array}{c}\text { Hauteur } \\
\text { Moyenne } \\
\mathrm{m}\end{array}$ & \\
\hline $\begin{array}{l}\mathrm{T} \ldots \ldots \\
\mathrm{TR} \ldots \\
\mathrm{PK} \\
\text { NPK } \ldots \\
\text { NPK }\end{array}$ & $\begin{array}{l}1,36 \\
1,36 \\
1,36 \\
1,38\end{array}$ & $\begin{array}{l}21,66 \\
22,31 \\
21,16 \\
21,87\end{array}$ & $\begin{array}{l}13,2 \\
13,3 \\
13,6 \\
13,5\end{array}$ & $\begin{array}{l}26,33 \\
27,15 \\
27,27 \\
28,61\end{array}$ & $\begin{array}{l}14,88 \\
15,02 \\
15,57 \\
15,35\end{array}$ & $\begin{array}{l}633 \\
661 \\
569 \\
627\end{array}$ \\
\hline
\end{tabular}

Ce sont uniquement les résultats de la période 1968-1971 qui seront commentés maintenant. Le tableau 9 donne les accroissements de surface terrière de 1968 à 1970 et de 1970 à 1971. L'effet de la fertilisation est très marqué en 1968-1970 (test $\mathrm{F}=22,02$ ); l'effet de PK par rapport à TR est significatif à $1 \%$, celui de NPK à $1 \%$, l'effet de NPK par rapport

TABLEAU $9-$ TABLE 9

Essai de l'Hermitage - Accroissements de surface terrière en $m^{2} / h a$ - Entre parenthèses les valeurs relatives par rapport au traitement TR.

L'Hermitage trial - Basal area increments $\left(m^{2} / h a\right)$ - Between brackets, relative values versus $\mathrm{TR}$ treatment.

\begin{tabular}{|c|c|c|c|}
\hline $\begin{array}{l}\text { Traitements } \\
\text { Treatments }\end{array}$ & $1968-1970$ & $1970-1971$ & $1968-1971$ \\
\hline 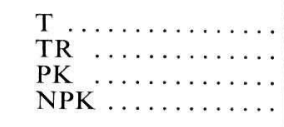 & $\begin{array}{ll}3,16 & (92) \\
3,44 & (100) \\
4,21 & (124) \\
4,75 & (138)\end{array}$ & $\begin{array}{l}1,50(108) \\
1,38(100) \\
1,88(136) \\
1,99(144)\end{array}$ & $\begin{array}{l}4,66(96) \\
4,84(100) \\
6,11(126) \\
6,75(139)\end{array}$ \\
\hline 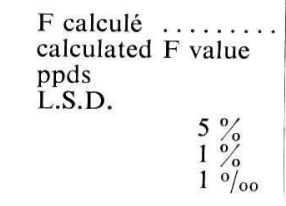 & $\begin{array}{l}0,47 \\
0,66 \\
0,94\end{array}$ & $\frac{0,45}{-}$ & $\begin{array}{l}0,71 \\
1,00 \\
1,41\end{array}$ \\
\hline $\begin{array}{l}\text { F théorique } \\
\text { Theoretical } F \text { value }\end{array}$ & $\begin{array}{l}5 \% \\
1 \% \\
1 \%\end{array}$ & $\begin{array}{r}3,49 \\
5,95 \\
10,80\end{array}$ & \\
\hline
\end{tabular}


à PK à $5 \%$ seulement. En 1970-71, l'action des engrais, notamment de l'engrais azoté, s'atténue : le test $\mathrm{F}$ tombe à 4,12 , l'effet de $\mathrm{PK}$ par rapport à TR n'est plus significatif qu'à $5 \%$, celui de NPK n'atteint pas tout à fait le seuil de $1 \%$, celui de NPK par rapport à PK n'est plus significatif.

Les volumes après éclaircie à la fin de 1968, considérés comme volumes initiaux pour cette deuxième phase de l'expérience, ont été calculés pour chaque placeau à partir de la surface terrière mesurée, des hauteurs moyennes déduites des hauteurs dominantes et de l'abaque de la table de production. Le tableau 10 donne les résultats et montre bien qu'à cette époque les volumes étaient pratiquement les mêmes dans tous les traitements. En novembre 1971, le volume final a été calculé à partir de la hauteur de bois fort et de la circonfé-

TABLEAU 10 TABLE 10

Essai de l'Hermitage - Volume en septembre 1968, après éclaircie (20 ans) - $m^{3} / h a$

L'Hermitage trial - Volume in september 1968, after thinning (20 years) - $m^{3} / h a$

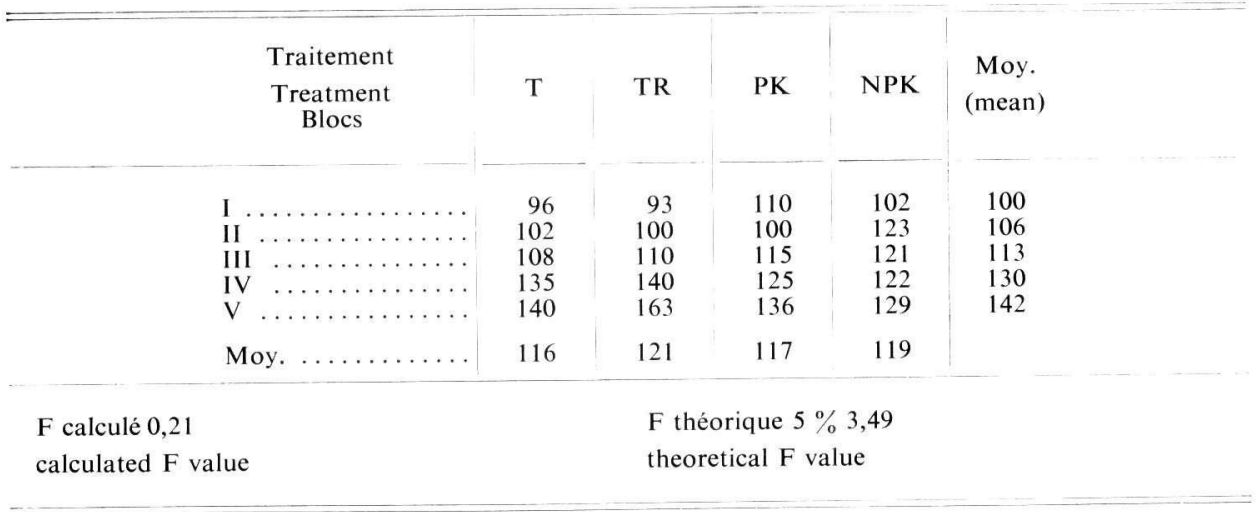

rence au milieu d'un échantillon représentatif des différentes catégories de circonférence. Le tableau 11 donne les résultats ainsi que l'accroissement 1968-71. L'ensemble des traitements est significatif à $1 \%$, l'effet de PK par rapport à TR est significatif à $5 \%$, celui de NPK par rapport à TR l'est à $1 \%$, celui de NPK par rapport à PK n'est pas significatif.

L'effet de la fertilisation est donc moins net qu'à Marcheprime, notamment celui de l'azote qui ne s'est fait sentir qu'en 1969 et 1970 . Peut-être est-ce dû au fait que le peuplement est plus jeune qu'à Marcheprime : d'une manière générale en effet, les peuplements jeunes réagissent davantage à l'acide phosphorique et les peuplements plus âgés davantage à l'azote.

Les gains d'accroissement courant en $\mathrm{m}^{3} / \mathrm{ha} / \mathrm{an}$ restent cependant élevés : $3,33 \mathrm{~m}^{3}$ pour PK et $5,26 \mathrm{~m}^{3}$ pour NPK alors que l'accroissement courant du témoin est de $16,57 \mathrm{~m}^{3}$.

Des analyses foliaires ont été effectuées sur des échantillons prélevés à la fin de 1971 sur tous les placeaux de cet essai, à raison de 2 arbres par placeau; les résultats moyens par traitement sont indiqués au tableau 12. Les teneurs en azote restent très basses, même si l'on tient compte des faibles exigences du Pin maritime à cet égard. Le traitement NPK se différencie faiblement du traitement PK sans toutefois atteindre un optimum qui se situe- 
TABLEAU 11 - TABLE 11

Essai de l'Hermitage - Volume en novembre 1971 - Accroissement de volume de 1968 à 1971 , en $\mathrm{m}^{3} / \mathrm{ha}$. L'Hermitage Trial - Volume in november 1971 - Volume increment from 1968 to 1971 ( $m^{3}$ par ha).

\begin{tabular}{|c|c|c|c|c|c|c|c|c|c|c|}
\hline \multirow{2}{*}{$\begin{array}{l}\text { Traitements } \\
\text { Treatments }\end{array}$} & \multicolumn{5}{|c|}{$\begin{array}{l}\text { Volume bois fort } 1971 \\
\text { Mercantable volume }\end{array}$} & \multicolumn{5}{|c|}{$\begin{array}{l}\text { Accroissement 1968-1971 } \\
\text { Volume increment }\end{array}$} \\
\hline & $\mathrm{T}$ & & TR & PK & NPK & $\mathrm{T}$ & TR & PK & NPK & Moy. \\
\hline \multicolumn{11}{|l|}{ Blocs } \\
\hline 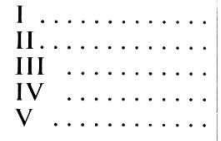 & $\begin{array}{l}140 \\
158 \\
157 \\
180 \\
191\end{array}$ & & $\begin{array}{l}140,2 \\
146,7 \\
155,7 \\
198,5 \\
223,4\end{array}$ & $\begin{array}{l}160,9 \\
165,1 \\
171,0 \\
187,1 \\
210,6\end{array}$ & $\begin{array}{l}156,0 \\
200,5 \\
195,8 \\
189,2 \\
192,8\end{array}$ & $\begin{array}{l}44,9 \\
56,8 \\
49,6 \\
45,8 \\
51,5\end{array}$ & $\begin{array}{l}47,2 \\
46,7 \\
45,7 \\
58,5 \\
60,4\end{array}$ & $\begin{array}{l}50,9 \\
65,1 \\
56,0 \\
62,1 \\
74,6\end{array}$ & $\begin{array}{l}54,0 \\
77,5 \\
74,8 \\
67,2 \\
63,8\end{array}$ & $\begin{array}{l}49,2 \\
61,5 \\
56,5 \\
58,4 \\
62,6\end{array}$ \\
\hline Moy. ......... & 165 & & 172,9 & 178,9 & 186,9 & 49,7 & 51,7 & 61,7 & 67,5 & 57,6 \\
\hline \multicolumn{2}{|c|}{$\begin{array}{l}\text { F calculé } \\
\text { calculated F value }\end{array}$} & 8,04 & 00 & \multicolumn{2}{|c|}{$\begin{array}{l}\text { ppds } 5 \% \\
\text { L.S.D. } 1 \%\end{array}$} & $\begin{array}{l}9,00 \\
12,63\end{array}$ & & & & \\
\hline \multicolumn{2}{|c|}{$\begin{array}{l}\text { F théorique } \\
\text { theoretical } F \text { value }\end{array}$} & $\begin{array}{l}5 \% \\
1 \% \\
1 \%\end{array}$ & $\begin{array}{r}3,4 \\
5,9 \\
10,80\end{array}$ & & & & & & & \\
\hline
\end{tabular}

rait vers $1,2 \%$. Ceci correspond bien à la constatation, déjà faite à propos de la production ligneuse, de la fugacité de l'action de l'engrais azoté. Pour le phosphore par contre, il y a une nette différence entre témoins et placeaux fertilisés qui montre l'action essentielle de cet élément. Il semble que le traitement NPK se différencie aussi des autres en ce qui concerne le potassium. Le traitement TR (sous-solage sans fertilisation) semble avoir entrainé une baisse d'absorption des éléments nutritifs; ce fait est à rapprocher du plus mauvais accroissement en surface terrière par rapport au témoin en 1970-1971. Or, rien de tel n'avait été constaté depuis 1962, année du sous-solage : peut-être faut-il voir là un effet de la sécheresse de 1971 qui expliquerait aussi la baisse d'action de la fertilisation au cours de cette année.

\section{TABLEAU 12 - TABLE 12}

Essai de l'Hermitage - Analyses foliaires fin 1971 (\% de la matière sèche).

L'Hermitage trial - Foliar analysises, end of 1971 (of \% dry matter).

\begin{tabular}{|c|c|c|c|c|}
\hline $\begin{array}{l}\text { Traitements } \\
\text { Treatmenis }\end{array}$ & $\mathrm{N}$ & $\mathrm{P}$ & $\mathrm{K}$ & $\mathrm{Ca}$ \\
\hline 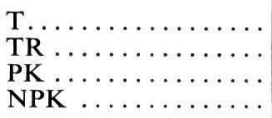 & $\begin{array}{l}0,92 \\
0,84 \\
0,84 \\
0,94\end{array}$ & $\begin{array}{l}0,068 \\
0,060 \\
0,093 \\
0,096\end{array}$ & $\begin{array}{l}0,49 \\
0,40 \\
0,49 \\
0,54\end{array}$ & $\begin{array}{l}0,16 \\
0,16 \\
0,18 \\
0,18\end{array}$ \\
\hline
\end{tabular}




\section{III. - DISCUSSION ET CONCLUSIONS}

Les deux essais de Marcheprime et de l'Hermitage comportent un certain nombre d'imperfections :

- petit nombre de traitements qui ne permet pas de connaître l'effet du potassium,

- application inadéquate des engrais au début de l'expérimentation,

- différences dans le procédé de mesure des volumes sur pied au début de l'expérience (à partir de la surface terrière et de la hauteur par un abaque) et à la fin (cube réel à partir de la hauteur et de la circonférence à mi-hauteur); pour avoir une idée de l'imprécision ainsi introduite, nous avons également calculé le volume en fin d'expérience par la méthode de la surface terrière; dans l'essai de Marcheprime, les différences d'accroissement courant par rapport au témoin s'établissent à $2 \mathrm{~m}^{3}$ pour $\mathrm{PK}$ (chiffre identique à celui calculé par la première méthode) et $2,7 \mathrm{~m}^{3}$ pour NPK (contre 3,4); à l'Hermitage, les différences par rapport à TR (témoin sous-solé) sont de $3,3 \mathrm{~m}^{3}$ pour PK (contre 3,4 pour la première méthode) et $4,4 \mathrm{~m}^{3}$ pour NPK (contre 5,3); c'est donc surtout l'effet de NPK dont l'appréciation paraît différer selon la méthode employée; on peut éventuellement voir dans ce fait l'effet d'un changement favorable du coefficient de forme sous l'influence de la fertilisation azotée comme cela été noté par certains auteurs sur diverses espèces de pins (Gooding, 1970, PeGG, 1966, Broerman, 1968); les volumes initiaux étant très peu différents dans la moyenne des placeaux destinés aux différents traitements, nous pensons d'ailleurs que si les accroissements en volume peuvent être entachés d'une certaine inexactitude, les différences d'accroissement qui ont été constatées entre traitements ne peuvent en être sérieusement affectées.

Ces deux essais montrent bien que les gains d'accroissement considérables permis par une fertilisation appliquée au moment du semis peuvent être obtenus aussi sur des peuplements adultes qui n'auraient pas reçu d'engrais au moment de leur création. Comme pour les semis, le phosphore joue le rôle essentiel; les analyses foliaires ne permettent pas de penser que le potassium a une action très importante. La fertilisation globale PK augmente l'accroissement de surface terrière de 25 à $35 \%$ et son effet est significatif à $1 \%$, parfois à $1 \%$ o L'azote apporté en supplément de PK a un rôle beaucoup moins net si on l'apprécie par différence entre les traitements NPK et PK; cette différence est cependant significative presque à $1 \%$ à Marcheprime. A l'Hermitage, le traitement NPK est très hautement significatif, tandis que le traitement PK ne diffère du témoin qu'à $1 \%$. On peut donc penser que l'azote, apporté en complément d'une fertilisation phosphatée de base, joue dans la fertilisation des peuplements adultes un rôle non négligeable. Grâce à lui, le gain d'accroissement en surface terrière peut atteindre 40 à $50 \%$. Rappelons d'ailleurs que, d'après MAUGÉ, des apports d'azote peuvent conduire, même sur des semis, à des gains de production importants ( 1 à $3 \mathrm{~m}^{3}$ ) à condition de ne pas être effectués avant la $6^{\mathrm{e}}$ année. L'augmentation d'accroissement courant en volume, encore qu'elle soit calculée de manière peu précise, comme nous l'avons signalé plus haut, est de l'ordre de $2 \mathrm{~m}^{3}$ par hectare pour la fertilisation PK et 3,5 $\mathrm{m}^{3}$ pour la fertilisation NPK. MAUGÉ arrive dans un de ses essais sur peuplement adulte, après une fertilisation NPK, à un gain d'accroissement courant de 4,2 $\mathrm{m}^{3}$ à 27 ans. Soulignons bien qu'il s'agit d'accroissement courant et non d'accroissement moyen (un gain d'accroissement moyen ne pourrait d'ailleurs avoir de sens que pour un peuplement fertilisé depuis sa création). 
On doit, bien sûr, se poser la question de la rentabilité de ces traitements. Dans l'essai de l'Hermitage, la durée d'expérimentation a été trop courte ( 3 ans entre l'application de la fertilisation « efficace » et la dernière mesure) pour que 1'on puisse faire un caclul intéressant. Pour Marcheprime où nous avons 10 ans de recul, le calcul a été effectué. Le coût de chaque application d'engrais a été évalué et capitalisé à $5 \%$ pendant la durée qui sépare l'application de la dernière mesure (fin 1971), soit 10 ans pour la première application de P et K, 6 ans pour la seconde, 9 ans, 8 ans, 7 ans et 4 ans pour les diverses applications d'azote. Le coût de l'opération s'établit ainsi, intérêts compris, à $916 \mathrm{~F} /$ ha pour la fertilisation $\mathrm{PK}, 832 \mathrm{~F}$ pour les applications d'azote et $1748 \mathrm{~F}$ pour la totalité des apports d'engrais. On peut, à partir de ces chiffres, calculer le prix de revient du $\mathrm{m}^{3}$ supplémentaire produit par $\mathrm{PK}$ $(44,50 \mathrm{~F})$ et par l'ensemble NPK $(50,80 \mathrm{~F})$. La rentabilité est donc suffisante quel que soit le système de fertilisation appliqué puisque les prix de revient calculés ci-dessus sont inférieurs ou égaux au prix de vente sur pied à l'âge qu'avaient atteint les pins en fin d'expérience (50 F environ).

Encore faut-il remarquer que l'estimation ci-dessus est la plus pessimiste possible, car on aurait très probablement pu obtenir les mêmes résultats techniques avec une seule application de $\mathrm{P}$ et $\mathrm{K}$, suivie de deux applications d'azote : par exemple, $200 \mathrm{~kg}$ de $\mathrm{P}_{2} \mathrm{O}_{5}$ et $100 \mathrm{~kg}$ de $\mathrm{K}_{2} \mathrm{O}$ en $1962,150 \mathrm{~kg}$ d'azote en 1963 et $150 \mathrm{~kg}$ d'azote à nouveau en 1968 . Il est d'autre part, vraisemblable que l'effet de la fertilisation se prolongerait au-delà des 10 ans sur lesquels ont porté les mesures. Dans ces conditions, en récoltant en 1977 et en supposant que sur ces 15 années, le gain dû à PK se limite à $1,5 \mathrm{~m}^{3} / \mathrm{ha} /$ an et celui dû à NPK à $2,5 \mathrm{~m}^{3} / \mathrm{ha} / \mathrm{an}$, les prix de revient du $\mathrm{m}^{3}$ supplémentaire s'établiraient respectivement à $27 \mathrm{~F}$ et $42 \mathrm{~F}$. Le bénéfice laissé par la fertilisation serait alors de $517 \mathrm{~F} /$ ha en 15 ans pour PK et $300 \mathrm{~F}$ pour NPK.

Cette rentabilité devrait d'ailleurs être diminuée de la baisse de densité et du rendement papetier, plus faibles de $10 \%$ environ pour les arbres fertilisés (JANIN, communication personnelle, Polge, 1969). Soulignons cependant que cette baisse ne s'applique pas au bois formé avant fertilisation.

Il serait d'ailleurs extrêmement utile, tant sur le plan technique que pour l'établissement de la rentabilité de l'opération, de savoir combien de temps peut durer l'action des différents types de fertilisation appliqués. Malheureusement, l'essai de l'Hermitage a été gravement endommagé par la tempête de février 1972 et ne se prêtera vraisemblablement plus à des interprétations suffisamment exactes.

Quoi qu'il en soit, nous pensons que ces premiers essais qui sont suivis par d'autres, pius complets, sont suffisamment positifs pour inciter les propriétaires forestiers des Landes à fertiliser les peuplements qui n'ont pas reçu d'engrais au moment du semis.

Bien que la rentabilité de l'opération diminue lorsque la durée qui s'écoule entre l'application des engrais et la récolte augmente, il semble que l'on puisse encore récupérer l'investissement en fertilisation en appliquant cette dernière 20 à 25 ans avant la coupe définitive. 


\section{REMERCIEMENTS}

Nous remercions très vivement Monsieur Good, gérant du Domaine de Marcheprime, qui nous a permis d'installer l'expérience dans la propriété.

Nous sommes également très reconnaissants à B. Lemorne et à l'équipe de l'Unité de Sylviculture de la Station de Bordeaux qui ont participé très activement à la détermination des volumes en fin d'essai.

\section{SUMMARY}

\section{RESULTS OF TWO MINERAL FERTILIZATION TRIALS ON MATURE MARITIME PINE} STANDS IN THE LANDES

On Landes podzols, south of Bordeaux, two fertilization trials were established in 1962 on mature maritime pines stands.

In the first one, at Marcheprime Forest Estate (age 39 years in 1962), two treatments PK et NPK were applied. During the ten years period of the trial, the total amounts applied were $380 \mathrm{~kg}$ of $\mathrm{P}_{2} \mathrm{O}_{5}$ and $212 \mathrm{~kg}$ of $\mathrm{K}_{2} \mathrm{O}$ per hectare, applied at two dates in 1962 and 1966, the first time in digging the fertilizer into localised sports; the second one, by broadcasting espace followed by a light soil disc ploughing. The nitrogen fertilizer was applied in 4 times, in 1963, 1964, 1965 and 1968 . The effect of fertilizers began to be obvious only from 1963 ahead. The volume increment from 1962 to 1971 was calculated : both treatments NPK and PK have a significant effect at the 0.001 level compared to check plots, but the effect of nitrogen (NPK versus PK) is significant at the 0.01 level only and seems to have ceased after 1968. The gain of mean current annual increment amounts to $2 \mathrm{~m}^{3} / \mathrm{ha} /$ year for PK treatment and to $3,4 \mathrm{~m}^{3}$ for NPK, the current annual increment for check plots being $7,7 \mathrm{~m}^{3} /$ ha/year.

In the Hermitage trial (14 years in 1962), the first fertilizer applications were made in 1962 by subsoil work followed by deep positioning phosphorus and potassium fertilizers, and broadcasting nitrogen. There were no effect. A new amount of $120 \mathrm{~kg}$ of $\mathrm{P}_{2} \mathrm{O}_{5}, 120 \mathrm{~kg}$ of $\mathrm{K}_{2} \mathrm{O}$ was broadcast in 1968 and lightly dug in by rotavatoring, and $100 \mathrm{~kg}$ of nitrogen were added in 1969 . The volume increment for the 1958-1971 period was calculated : the effect of PK treatment is significant at the 0,05 level, of NPK at 0,01 . The effect of nitrogen NPK versus PK is not significant. The mean current annual increment for check plots is $16,6 \mathrm{~m}^{3} / \mathrm{ha} /$ year, the gain due to $\mathrm{PK}$ is $3,3 \mathrm{~m}^{3}$ and to NPK, $5,2 \mathrm{~m}^{3}$.

In conclusion, it appears that mature Landes maritime pines react to fertilization as well as young seedlings do. Nitrogen, the effect of which on seedlings is generally low, seems to play here an interesting role. Economic calculations show that, if invested funds are capitalized at $5 \%$ rate, fertilization can give a benefit of about 40 to $50 \mathrm{~F}$ per hectare and per year.

At Marcheprime, where gum tapping was done, fertilization had no effect on gum yield.

\section{ZUSAMMENFASSUNG}

\section{ERGEBNISSE VON ZWEI DÜNGUNGSVERSUCHEN MIT ERWACHSNEN SEESTRANDKIEFERN IN DEN « LANDES »}

In den « Landes » im Süden von Bordeaux wurden zwei Forstdüngungsversuche in Baum- und Stangen-hölzern von Seestrandkiefer (Pinus pinaster Ait.) auf Podzolböden im Jahre 1962 angelegt.

Im Versuch " Marcheprime » (Bestandesalter zu Versuchsbeginn : 39 Jahre) wurden zwei Behandlungen, PK und NPK untersucht. Während der zehnjährigen Versuchsdauer wurden insgesamt $380 \mathrm{Kg} \mathrm{P}_{2} \mathrm{O}_{5}$ und $212 \mathrm{~kg} \mathrm{~K}_{2} \mathrm{O}$ in zwei Teilen (1962 und 1966) ausgebracht, wobei die Dünger das 
erste Mal plätzeweise in den Boden eingebracht wurden während die zweite Düngung durch Streuung und nachfolgende leichte Bodenbearbeitung erfolgte; die ingesamt $280 \mathrm{~kg}$ Strickstoff wurden in den Jahren 1963, 1964, 1965 und 1968 gegeben. Eine Düngerwirkung machte sich erst dem Jahre 1963 bemerkbar. Es wurde der Volumenzuwachs für die Periode von 1962-1971 berechnet. Die beiden Behandlungen (PK und NPK) zeigen bezüglich der Nullfäche einen hochsignifikanten Unterschied $(P=0,001)$; die Stickstoffwirkung (Unterschied zwischen NPK und PK) ist gleichfalls signifikant $(\mathbf{P}=\mathbf{0}, 01)$, scheint jedoch nach dem Jahre 1968, nachgelassen zu haben. Der mittlere jahrliche Zuwachsgewinn beträgt 2,0 fm/ha (PK) und 3,4 fm/ha (NPK); der mittlere laudenfe Zuwachs der Nullfläche während der Versuchsperiode ergab sich mit 7,7 fm pro Hektar und Jahr.

Im Versuch « L'Hermitage » (Alter 14 zu Versuchsbeginn) wurde die erste Düngergabe (P und K) nach Tiefpflügung tief in den Boden eingebracht, während der Stickstoff oberflächlich gestreut wurde. Diese erste Ausbringung zeigte keinen messbaren Erfolg. Im Jahre 1968 wurden neuerlich $120 \mathrm{~kg} \mathrm{P}_{2} \mathrm{O}_{5}$ und $120 \mathrm{~kg} \mathrm{~K}_{2} \mathrm{O}$ ausgestreut und durch eine Rotavatorbehandlung geringfügig mit dem Oberboden vermischt; desweiteren wurden $1969100 \mathrm{~kg}$ Stickstoff gestreut. Es wurde der Massenzuwachs für die Periode 1968-1971 berechnet. Es bestehen signifikante Unterschiede zwischen der Nullfläche und PK $(P=0,05)$ sowie NPK $(P=0,01)$; die Stickstoffwirkung ist nicht gesichert. Der mittlere laufende Zuwachs der Nullfä̈che während der Versuchsperiode betrug $16.6 \mathrm{fm}$ pro Hektar und Jahr. Es ergibt sich ein jährlicher Zuwachsgewinn von $3.3 \mathrm{fm} / \mathrm{ha}$ (PK) bzw. 5,2 fm/ha (NPK).

Abschliessend kann festgestellt werden, dass auch Baum- und Stangenhölzer der Seestrandkiefer und nicht nur die Kulturen auf eine Düngung günstig ansprechen. Während die Kulturen auf eine Stickstoffdüngung im allgemeinen nur gering ansprechen, scheint die Stickstoffdüngung in älteren Beständen von Bedeutung zu sein. Eine Berechnung der Wirtschäftlichkeit zeigt, dass bei einer fünfprozentigen Kapitalisierung der Kosten mit einem jährlichen Gewinn von 45 bis 50 Franken pro Hektar gerechnet werden kann.

Im Versuch « Marcheprime » bei dem einer der beiden Bestände geharzt wurde, konnte kein Einfluss der Düngung auf die Harznutzung festgestellt werden.

\section{RÉFÉRENCES BIBLIOGRAPHIQUES}

Gooding (J.-M.). Effects of fertilization on crown, stem and wood properties of Slash Pine. Abstract of Thesis - School of Forestry - Gainesville (U.S.A.), 1970.

Guinaudeau (J.), Illy (G.), Mauge (J.-P.), Dumas (F.) - Essai de fertilisation minérale sur Pin maritime à Mimizan (Landes). Ann. Ec. nat. des Eaux et Forêts et Stat. Rech. Exper. forest. 20 (1), 1963. JANIN (G.), Nouveau critère d'homogénéité du bois au point de vue des propriétés physiques : le diagramme
polaire différentiel des rendements. Ann. Sci. forest (sous presse).

MAUGÉ (J.-P.), Études et expérimentations sur pin maritime 1965-1970, A.F.O.C.E.L., 1972. POLGE (H), Influence de la fertilisation sur la qualité du bois de Pin maritime. Ann. Sci. forest 26 (1) (45-46),
1969. 